\title{
High-pressure Luminescence Study of GdOCl:Eu Phosphor
}

\author{
Shenkin Liu, Yuanbin Chi*, Xuyi Zhao, Lizhong Wang*, and Guangtian Zou* \\ Changchun Institute of Physics, Academia Sinica, Changchun 130021, China \\ * National Laboratory of Superhard Materials, Jilin University, Changchun 130023, China
}

\begin{abstract}
The emission spectra of $\mathrm{GdOCl}$ : Eu have been measured at high pressures up to $10 \mathrm{GPa}$ and at room temperature to examine the pressure effect on the energy levels of $\mathrm{Eu}^{3+}$ in the crystal. About 60 spectral lines have been measured at various pressures. These lines slowly shift to the red at rates $3-9 \mathrm{~cm}^{-1} / \mathrm{GPa}$. On the basis of experimental data the pressure dependences of 24 Stark levels belonging to the ${ }^{5} \mathrm{D}_{0-2}$ and ${ }^{7} \mathrm{~F}_{0-5}$ multiplets have been determined. By fitting experimental levels the crystal field parameters and free ion parameters have been found out. The crystal field strength parameter $S$, Slater integral $F_{2}$ and spin-orbit coupling parameter $\zeta$ decrease with pressurization in some degree.

[high pressure luminescence, $\mathrm{GdOCl}$; Eu, energy level, crystal field effect]
\end{abstract}

\section{Introduction}

The rare earth oxyhalides (REOX) constitute a versatile matrix system, widely isomorphous but displaying considerable changes in the coordination of the $\mathrm{RE}^{3 \div}$ ion. This versatility provides an excellent opportunity to study the variation of the crystal field (CF) effects along both the rare earth and the halide series. On the other hand, the $\mathrm{Eu}^{3+}$ ion gives a convenient approach to the study of the interaction between the central ions and their crystalline environment because of the particular features of its energy level scheme and the easier interpretation of its spectrum. Therefore $\mathrm{Eu}^{3+}$ activated rare earth oxyhalides have received a lot of scientific attention $[1-3]$ and become an important object in the studying of the CF effects.

High pressure can continuously decrease the interatomic distance in crystals and is widely used in studying the physical and chemical properties of crystals. We had studied the luminescence properties of $\mathrm{LaOCl}: \mathrm{Eu}$, $\mathrm{LaOBr}: \mathrm{Eu}$ and $\mathrm{GdOBr}: \mathrm{Eu}$ under high pressure $[4-6]$ in order to examine the effect of crystal field on the energy level scheme of the RE ions in continuous contraction of the interatomic distance. The details have been reported in our previous papers $[4-6]$. In these studies we observed some interesting phenomena. Firstly, the splitting of the two Stark levels of the ${ }^{7} \mathrm{~F}_{1}$ multiplet decreases with increasing pressure. Secondly, the crystal field strength parameters $S$ of these samples decrease in pressurization process although a decrease in the interatomic distance leads to an increase in interaction between atoms. And the free ion parameters $F_{k}$ and $\zeta$ also decrease with the pressure increase.

In the present work, we studied the luminescence of $\mathrm{GdOCl}:$ Eu under high pressure in order to know if this sample has the above mentioned properties.

\section{Experimental}

The polycrystalline $\mathrm{GdOCl}: \mathrm{Eu}$ sample used in this work was prepared by a solid state reaction between the corresponding rare earth oxides and ammonium chloride at high temperature. The nominal concentration of Eu in the sample is $5 \mathrm{~mol}$. \%. The sample was routinely checked by $x$-ray powder diffraction. The high pressure was generated with a gasketed diamond anvil cell and calibrated by the ruby fluorescence method. The wellknown 4:1 methanol-ethanol mixture was used as pressure transmitting medium. The $465.8 \mathrm{~nm}$ line of an argon ion laser was used to pump the sample and a Spex-
1403 Raman Spectrometer to record the luminescence of the sample. The spectrum measured in the range from $21430 \mathrm{~cm}^{-1}$ to $13000 \mathrm{~cm}^{-1}$ nearly includes all the emissions from ${ }^{5} \mathrm{D}_{0-2} \rightarrow{ }^{7} \mathrm{~F}_{0-5}$ transitions. About 60 spectral lines were recorded at room temperature and high pressures up to $10 \mathrm{GPa}$. Their assignment was carried out according to Hölsä's work [1].

\section{Results and Discussion}

The spectral lines measured in the pressure range of this work shift to the red with pressurization. The red shift rates are between $3 \mathrm{~cm}^{-1} / \mathrm{GPa}$ and $9 \mathrm{~cm}^{-1} / \mathrm{GPa}$. This phenomenon shows that the CF levels of the ${ }^{5} D_{0-2}$ multiplets lower more rapidly than those of the ${ }^{7} F_{0-5}$ multiplets. On the basis of the peak positions of the lines measured at various pressures the dependence of the CF levels on the pressure was determined and plotted in Fig. 1. From the figure it can be seen that all the levels slowly change with increasing pressure. This phenomenon represents the fact that $4 \mathrm{f}$ electrons shielded by $5 \mathrm{~S}^{2} 5 \mathrm{P}^{6}$ electronic shell are insensitive to outside pressure effects. The two levels of ${ }^{7} F_{1}$ close to each other with pressurization, as might be expected.

After knowing the change in CF levels, it is possible to study the effects of pressure on the interatomic and intra-atomic interactions for $\mathrm{Eu}^{3+}$ in $\mathrm{GdOCl}: \mathrm{Eu}$. As we know, for an $\mathrm{RE}^{3+}$ ion in a crystal the Hamiltonian $H$ can be written as

$$
H=H_{s}+H_{c},
$$

where: $H_{f}$ is the free ion Hamiltonian describing the interaction inside the $\mathrm{RE}^{3+}$ ion and $H_{C}$ the CF Hamiltonian representing the interaction between the $R^{3-}$ ion and its crystalline environment. The $\mathrm{Eu}^{3+}$ ion in the $\mathrm{GdOCl}$ : Eu mainly occupies the site with $C_{4 v}$ symmetry and its $H_{C}$ can be expressed as

$$
\begin{aligned}
H_{C}= & B_{0}^{2} C_{0}^{2}+B_{0}^{4} C_{0}^{4}+B_{4}^{4}\left(C_{-4}^{4}+C_{4}^{4}\right) \\
& +B_{0}^{6} C_{0}^{6}+B_{4}^{6}\left(C_{-4}^{6}+C_{4}^{6}\right) .
\end{aligned}
$$

In the expression $C_{q}^{k}$ are the tensor operators associated with spherical harmonics by

$$
C_{q}^{k}=[4 \pi /(2 k+1)]^{1 / 2} \sum_{i} Y_{q}^{k}\left(\theta_{i}, \varphi_{i}\right)
$$



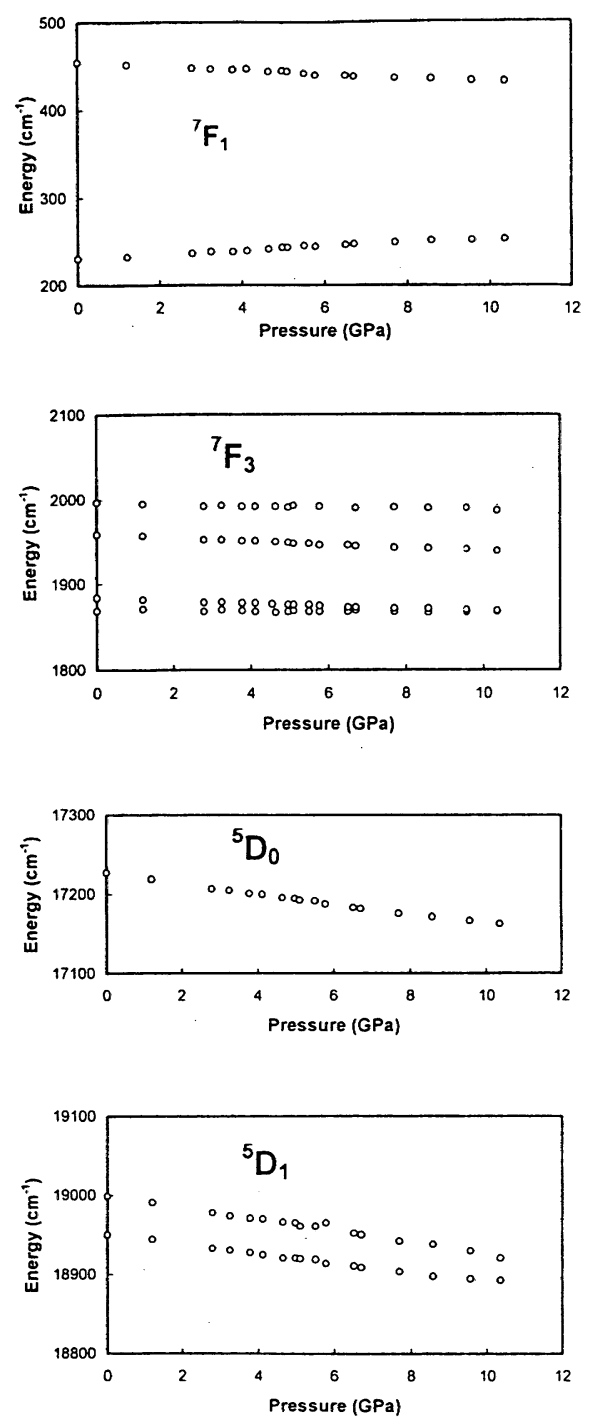
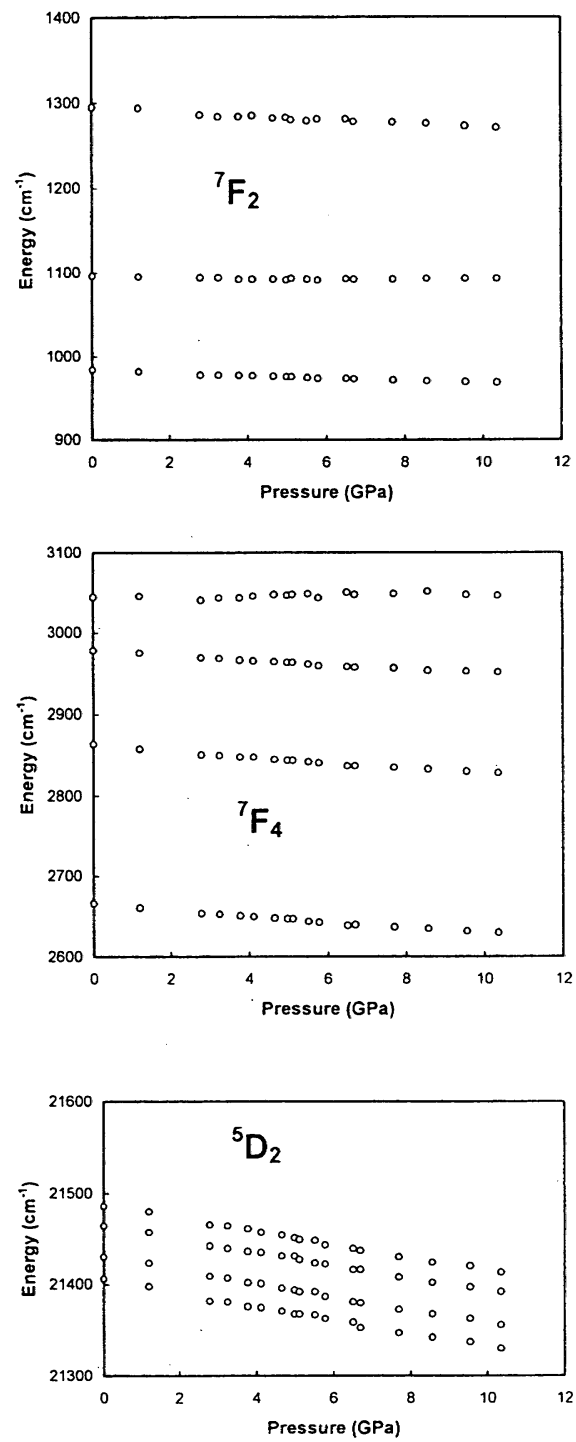

Fig. 1. Effect of pressure on the levels of the ${ }^{7} \mathrm{~F}_{1-4}$ and ${ }^{5} \mathrm{D}_{0-2}$ multiplets.

and $B_{q}^{k}$ the CF parameters to be determined. There are $24 \mathrm{CF}$ levels available to derive these CF parameters. In the calculation the ${ }^{7} \mathrm{~F}$ and ${ }^{5} \mathrm{D}$ manifolds have been considered. In the constructing of the matrix of $H_{C}$ the intermediate coupling wave functions and J-mixing were taken into account. By fitting the calculated levels to the observed ones the five CF parameters were obtained in the least squares calculation. The result is given in Table 1. The CF strength parameter and the rms deviation $\sigma$ are also given in the same table. The strength parameter $S$, defined by Chang $[7]$, can be derived from the $B_{q}^{k}$ set :

$$
S=\left\{\frac{1}{3} \sum_{k} \frac{1}{2 k+1}\left[\left(B_{0}^{k}\right)^{2}+2 \sum_{q>0}\left|B_{q}^{k}\right|^{2}\right]\right\}^{1 / 2} .
$$

$S$ is an invariant under arbitrary rotation of the crystal lattice and also a quantitative measure of the $\mathrm{CF}$ strength. From the table it can be seen that $S$ decreases with increasing pressure, which is similar to the situation in $\mathrm{LaOCl}: \mathrm{Eu}, \mathrm{LaOBr}: \mathrm{Eu}$ and $\mathrm{GdOBr}: \mathrm{Eu}[4-6]$.

As we know pressure affects not only the interaction between the RE ion and its crystal environment, but also the interaction inside the $\mathrm{RE}$ ion. In the first approximation

$$
\begin{aligned}
H_{f}= & \sum_{k} f^{k} F_{k}+\zeta \sum_{i} s_{i} \cdot l_{i} \\
& +\left[\alpha L(L+1)+\beta G\left(G_{2}\right)+\gamma G\left(R_{7}\right)\right],
\end{aligned}
$$


where the first term stands for the $4 \mathrm{f}$ electron Coulomb repulsion, the second term represents the spin-orbit interaction and the last term expresses the configuration interaction. $F_{k}(k=2,4,6)$ are the Slater parameters and $f^{k}$ the corresponding coeffecients. $\zeta$ is the spin-orbit couping parameter, $l_{i}$ and $s_{i}$ are, respectively, the orbital and spin angular monentum of the ith $4 \mathrm{f}$ electron. $\alpha, \beta$ and $\gamma$ are the configuration interaction parameters, $L$ is the total orbital angular momentum, $G\left(G_{2}\right)$ and $G\left(R_{7}\right)$ are the eigenvalues of Casimir's operators for the Lie groups $G_{2}$ and $R_{7}$. To minimize the number of free parameters, we express $F_{4}$ and $F_{6}$ with $F_{2}$ by fixing the ratios of $F_{4} / F_{2}=0.1484$ and $F_{6} / F_{2}=0.0161$. On the basis of the experimental values of the ${ }^{7} \mathrm{~F}_{0-4}$ and ${ }^{5} \mathrm{D}_{0-2}$ multplet barycenters, the values of $F_{2}$ and $\zeta$ at various pressures have been obtained in the least squares refining calculations and are plotted in Fig. 2. In the pressure range of the present work the values of $F_{2}$ and $\zeta$ decrease by $0.38 \%$ and $0.44 \%$, respectively, which is similar to the situation in $\mathrm{GdOBr}: \mathrm{Eu}$ [6].

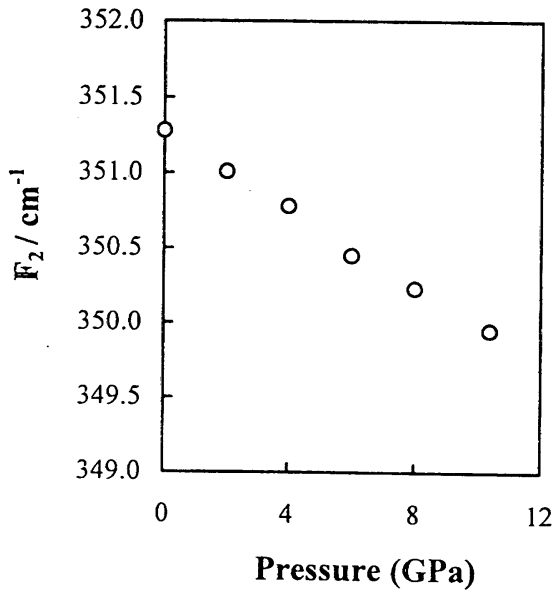

Table 1 CF parameters, CF strength parameters and rms deviations (in $\mathrm{cm}^{-1}$ ) at pressures (in $\mathrm{GPa}$ ).

\begin{tabular}{ccccccc}
\hline$P$ & 0 & 2 & 4 & 6 & 8 & 10 \\
$B_{0}^{2}$ & -946 & -908 & -858 & -812 & -786 & -761 \\
$B_{0}^{4}$ & -685 & -676 & -668 & -698 & -738 & -758 \\
$B_{4}^{4}$ & 887 & 896 & 900 & 887 & 869 & 869 \\
$B_{0}^{6}$ & 1039 & 1113 & 1111 & 1140 & 1221 & 1224 \\
$B_{4}^{6}$ & 386 & 381 & 416 & 428 & 397 & 403 \\
$S$ & 413 & 413 & 408 & 404 & 404 & 403 \\
$\sigma$ & 11 & 12 & 11 & 11 & 11 & 12 \\
\hline
\end{tabular}

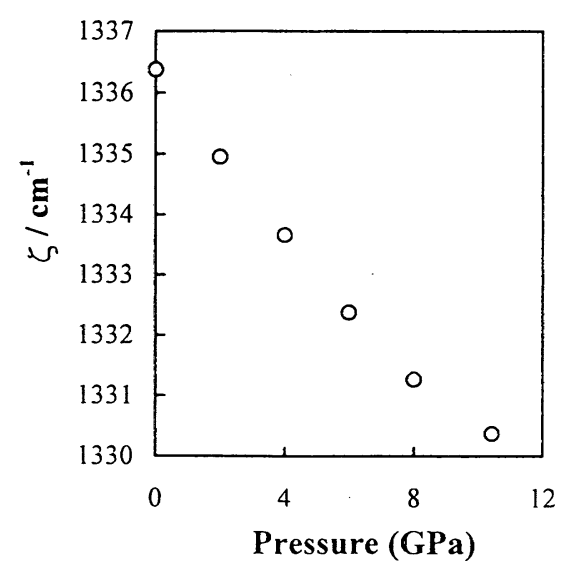

Fig. 2. Dependences of the Slater parameter $F_{2}$ and the spin-orbit coupling parameter $\zeta$.

\section{Acknowledgment}

This work has been supported by the National Natural Science Foundation of China.

\section{References}

[1] J. Hölsä, P. Porcher, J. chem. Phys. , 75, 2108 (1981).

[2] J. Hölsä, P. Porcher, J. Chem. Phys, , 76, 2790 (1982).

[3] J. Hölsä, E. Kestilä, J. Alloys Comp. , 225, 89 (1995).

[4] Chi Yuanbin, Liu Shenxin, Shen Wufu, Wang
Lizhong, and Zou Guangtian, Physica, 139\&140 $B, 555$ (1986).

[5] Chi Yuanbin, Liu Shenxin, Wang Qiuping, Wang Lizhong, and Zou Guangtian, High Press. Res., 3, 150 (1990).

[6] Chi Yuanbin, Liu Shenxin, Li Hongnian, Zhao Xuyi, and Wang Lizhong, J. Alloys Comp. , 256, 1 (1997).

[7] N. C. Chang, J. B. Gruber, R. P. Leavitt, C. A. Morrison, J. Chem. Phys., 76, 3877 (1982). 